\title{
Construir la ciudad: Un paradigma de gestión urbana. Propuesta de un método para viabilizar planes urbanos
}

\author{
Ferrer y Arroyo, Mercedes * \\ Avila de Montero, Nancy **
}

\section{Resumen}

La preocupación por la gestión surge del cuestionamiento a la validez de los planes urbanos para imprimir racionalidad a los procesos de transformación urbana o para ordenar las ciudades y, generar cambios en la calidad de vida y en el tejido social. El objetivo de este trabajo es exponer la metodologia utilizada en la elaboración del Plan de Gestión de Tamare (Estado Zulia, Venezuela), el cual se concibe como plan de participación y gestión que integra la gestión urbana con la intervención social, a través del marketing urbano, para generar nuevos métodos y prácticas urbanas que viabilizan el proceso de construcción de la ciudad en el siglo XXI. La metodología utilizada para lograr la viabilidad política, institucional, social y financiera del Plan Maestro de Desarrollo Urbano de Tamare se estructura en tres Momentos, el Momento 1: Construcción del Mensaje para explicar el Plan Maestro, Momento 2: Organización de los Actores y diseño de las estructuras organizativas que operativizan la viabilización y ejecución del Plan y el Momento 3: Construcción del Consenso o estrategia operativa. Este último momento se desarrolla en tres etapas, Etapa 1: Compromiso de Participación, Etapa 2: Marketing de Tamare y Etapa 3: Corresponsabilidad en la Gestión. En esta última etapa se firma el Convenio de Gestión

Recibido: 99-01-11, Aceptado: 99-10-19

* Arquitecto. Maestria en Planificación Urbana. Profesora Titular de la Facultad de Arquitectura y Diseño de LUZ. Coordinadora del Programa de Especialización en Gestión Urbana. Directora de Extensión. E.mail: mferrer@luz.ve, mferrer@cantv net

** Arquitecto. Maestría en Planificación Regional. Profesora Titular de la Facultad de Arquitectura y Diseño de LUZ. Membro del Comité Académico del Programa de Especialización en Gestión Urbana y de la Maestría en Planificación Integral para el Desarrollo del Turismo. Directora de Planificación Físicà de LUZ. 
que concreta el compromiso de los diferentes actores con el Plan Maestro de Tamare. La metodología planteada promueve una nueva concepción de la planificación urbana como proceso de negociación y gestión coordinada y continua entre diversos actores, públicos y privados, que permite la selección consensuada de vías para solucionar los problemas percibidos en el uso cotidiano de las ciudades y, movilizar la confianza activa, a través de la participación democrática y sinérgica de los actores, para que sean corresponsables en la ejecución del Plan Maestro.

Palabras clave: Plan de gestión, participación, convenio de gestión, metodologia, marketing urbano.

\section{To Build the City: A Paradigm of Urban Management. The Proposal of a Method to Make Urban Plans Viable}

\section{Abstract}

The problem of urban management arises from the questioning as to the validity of urban plans in order to assure rationality in the urban transformation processes or in the re-ordering of cities and, to generate changes in the quality of life and in the urban social fabric. The objective of this paper is to explain the methodology used in the elaboration of a management plan for Tamare (Zulia State, Venezuela). This plan was conceived as a participatory management plan that integrated urban management with social intervention, through urban marketing, in order to generate new urban methods and practices and to make possible the construction of this city in the XXI Century. The methodology applied to achieve the political, institutional, social and financial viability of the Master Plan for the Urban Development of Tamare was structured in three time periods. Moment 1 was the construction of the message explaining the Master Plan. Moment 2 was the organization of the actors and the design of the organizational structures that would oversee the viability and execution of the plan. Moment 3 was the building of consensus and/or operational strategy. The third moment was developed in three stages; Stage 1: agreement to participate; Stage 2:the marketing of Tamare; and Stage 3: co-responsibility in its realization. In this third stage, a management agreement was signed by all the different actors in the Master Plan for Tamare, in order to formalize their commitments. This methodology promoted a new conception of urban planning as a process of coordinated and continuous negotiation and management between diverse actors both public and private, which permits the selection by consensus of manners to solve problems that are perceived daily in city life, and the mobilization of active confidence, through democratic and synergetic participation of all actors, permitting them to be co-responsible in carrying out the Master Plan.

Key words: Management plan, participation, management agreement, methodology, urban marketing. 


\section{Introducción}

Los planes urbanos elaborados durante los últimos veinte años han propiciado una opinión generalizada que cuestiona su validez para imprimir racionalidad a los procesos de transformación urbana o para ordenar las ciudades y, generar cambios, en la calidad de vida y en el tejido social. La preocupación por la gestión, es decir, por la aplicabilidad posterior de las decisiones de los planes urbanos se relaciona con el debate urbanistico de los últimos años el cual considera que no hay plan bueno sin adecuada ejecución posterior (Ferrer, 1993 y 1995). Asumiendo esta premisa, Petróleos de Venezuela (PDVSA) solicitó a la Facultad de Arquitectura de la Universidad del Zulia la elaboración del Plan de Gestión de Tamare. Este Plan se realizó en dos etapas. En primera etapa, identificada como Relaciones con la Comunidad (Ferrery Avila, 1997), se diseñó el Plan de Gestión $y$, en la segunda denominada, Estrategia de Intervención Social para viabilizar el Plan Maestro de Tamare, (Martínez et al, 1997), se ejecutó dicho Plan. Este artículo describe la primera etapa.

Tamare es una urbanización petrolera abierta (integrada a Ciudad Ojeda), habitada por empleados activos $y$, PDVSA, localizada en la periferia norte de Ciudad Ojeda, capital del Municipio Lagunillas de la de la Costa Oriental del Lago de Maracaibo (COLM) del Estado Zulia. Ocupa un área aproximada de $315 \mathrm{Ha}$. La Avenida Intercomunal divide la Urbanización en dos lotes, el lote Este ya urbanizado y casi totalmente ocupado y el lote Oeste, que colinda con el
Lago de Maracaibo, aun no desarrollado. Tamare tiene una posición geográfica estratégica en la COLM, calidad de vida y ambiental que se refleja en la dotación de senicios de equipamiento e infraestructura por encima muchas veces de la norma, que permiten posicionarla como un "hábitat de calidad en la COLM". Tamare representa una forma de hacer ciudad típica de los campamentos petroleros de la Costa Oriental del Lago que es necesario consolidar.

El Plan de Gestión de Tamare se concibe como plan de participación y gestión que integra la gestión urbana con la intervención social, a través del marketing urbano, para generar nuevos métodos y prácticas urbanas que viabilizan el proceso de construcción de la ciudad. El objeto de este Plan es lograr la viabilidad política, institucional, social - cultural y financiera del Plan Maestro de Desarrollo Urbano de Tamare. El Plan de Gestión asume la planificación urbana como proceso de negociación y gestión coordinada y continua entre diversos actores, públicos y privados a fin de seleccionar, por consenso, vías para solucionar los problemas percibidos en el uso cotidiano de las ciudades. Movilizar la confianza activa, a través de la participación democrática y sinérgica de los actores, para que sean corresponsables en la ejecución del Plan Maestro, constituye la garantía de éxito del Plan de Gestión propuesto. El proceso de viabilización tiene dos objetivos básicos, compatibilizar las expectativas de los actores sociales con las propuestas del PLAN MAESTRO DE DESARROLLO URBANO DE TAMARE y establecer patrones de conducta nuevos para 
inducir a la toma de decisión en forma consensuada.

En el PLAN de GESTION de TAMARE se propone un método o modelo de gestión para planificar y ejecutar las acciones necesarias para viabilizar planes urbanos ex-post, en este caso, el Plan Maestro de Desarrollo Urbano de Tamare y se estructura en tres Momentos, el Momento 1: Construcción del Mensaje para explicar - viabilizando el Plan Maestro, Momento 2: Organización de los Actores y diseñó de dos estructuras organizativas, una temporal y otra permanente, que operativizan la viabilización y ejecución del Plan, el Comité de Gestión $y$, la Fundación para el desarrollo de la Urbanización Tamare (FUNDATAMARE) respectivamente. El Momento 3, Construcción del Consenso (estrategia operativa) se desarrolla en tres etapas, Etapa 1: Compromiso de Participación, Etapa 2: Marketing de Tamare y Etapa 3: Corresponsabilidad en la Gestión. En esta última etapa se instala FUNDATAMARE y se firma el Convenio de Gestión que concreta el compromiso colectivo, de los diferentes actores con el Plan Maestro de Tamare. La ejecución del Convenio de Ges. tión garantiza el mantenimiento y consolidación de la calidad de vida en Tamare objetivo fundamental del Plan de Gestión de Tamare (Ferrer et al, 1997). El objetivo de este trabajo es exponer la metodología utilizada para la elaboración del Plan de Gestión en referencia.

Sobre la base de las consideraciones anteriores el artículo se estructura en dos partes. En la primera se presenta el encuadre teórico y se conceptualiza la gestión urbana y, en la segunda parte, proponemos una metodología para viabilizar planes urbanos ex-post.

\section{Encuadre teórico sobre la gestión urbana}

La propuesta de un método para que los agentes urbanos asuman y se comprometan en el proceso de ejecución de los planes urbanos surge de la necesidad de superar los problemas que viene enfrentando la planificación urbana en Venezuela. Los planes urbanos pocas veces se ejecutan, a pesar de que una vez aprobados se constituyen en ley, como lo establece el artículo 42, parágrafos primero y segundo, de la Ley Orgánica de Ordenación Urbanística, LOOU, (1988). Entre las causas de esta situación están: 1. la falta de participación ciudadana por una parte del desconocimiento de las Leyes Orgánicas de Régimen Municipal y, de Ordenación Urbanística, que facultan a los ciudadanos a participar en el proceso de formulación y gestión de los planes. La LOOU, en su artículo 38 establece que, "los planes de desarrollo urbano local, una vez elaborados, serán sometidos a un proceso de información y consultas públicas por un periodo de 60 días continuos $y$, durante ese lapso, los interesados podrán hacer las observaciones que estimen oportunas o convenientes". En Venezuela, el proceso de participación y consulta pública, según la LOOU, se realiza ex-post, en consecuencia el plan no es el resultado de un proceso o proyecto urbano consensuado entre los diferentes actores urbanos. Por otra parte, la falta de participación esta vinculada a la inexperiencia y poca tradición 
participativa de los ciudadanos, el desconocimiento de los Alcaldes y de los ciudadanos, de la utilidad y el potencial de los planes urbanos, para mejorar la calidad de vida en las ciudades. 2. existe contradicción entre la flexibilidad que requiere el proceso político de toma decisión para abordar problemas complejos en un entorno cambiante con alto grado de incertidumbre y, la rigidez metodológica, de los planes urbanos.

Para superar estos problemas, en los últimos diez años se han propuesto diferentes modelos de gestión que buscan viabilizar los planes urbanos (Ayuntamiento de Madrid, 1996; Ferrer, 1993 y 1995) y, se ha iniciado una nueva práctica urbana en muchos países, a partir de la formulación y gestión de planes estratégicos de ciudades, como Madrid, Bilbao, Barcelona en España, Caracas y, en Tamare el Plan de Gestión que se presenta en este trabajo, en los cuales participan, desde su inicio, los diferentes actores urbanos. Es decir, el plan se construye a partir de una consulta pública permanente constituyéndose así en un proyecto consensuado de ciudad. En este sentido, coincidimos con Melo Moya (1995) coincide con nuestra propuesta al plantear que, "elMunicipio necesita incorporar en su gestión, una estrategia que oriente las ideas centrales que direccionan la intervención social, económica y cultural en el territorio comunal. Este conjunto de ideas fuerza deben plasmarse en la cotidianeidad de la gestión, alimentando las políticas municipales de desarrollo local".

\subsection{Planificación estratégica de ciudades}

La fabrica física y el tejido socioeconómico de nuestras ciudades tienen dificultades para adaptarse a los cambios geopolíticos, económicos-sociales, tecnológicos y administrativos. Ante esta situación cabe preguntarse ¿ son los actuales instrumentos de planificación y gestión urbana válidos para responder a los cambios del entorno? ¿Hacia donde debe encaminarse la planificación y gestión urbana para impulsar la competitividad y habitabilidad de las ciudades? Para viabilizar las operaciones reestructuradoras del tejido físico, económico y social de las ciudades se necesita, según Fernández Gúell (1997), un proyecto estratégico que cumpla por lo menos con dos objetivos: "articular las acciones sectoriales en un programa global y estimular al conjunto de la sociedad para lograr el horizonte o visión definida". En este sentido y, según Fernández Gúell, (1997: 54), la planificación estratégica de ciudades puede definirse como, "una forma sistemática de manejar el cambio y de crear el mejor futuro para la ciudad o, un proceso creativo que sienta las bases de una actuación integrada a largo plazo, establece un sistema continuo de toma de decisión que comporta riesgos, identifica cursos de acción especificos, formula indicadores de seguimiento sobre los resultados e involucra a los agentes sociales y económicos locales a lo largo de todo el proceso". Gran parte de estos conceptos proceden de la planificación empresarial, debido al 
paralelismo existente entre el sistema empresa y el sistema ciudad (Cuadro 1).

Para este autor la planificación estratégica de ciudades tiene las características siguientes: aporta una visión de largo plazo de carácter plurilegislativo, es decir, al ser un proyecto asumido por toda la comunidad se desarrolla a lo largo de varias legislaturas con independencia de los cambios políticos; considera el entorno; identifica las ventajas competitivas y comparativas de la ciudad; aporta una visión integral de la realidad urbana supera la visión sectorial y asume como objetivo último mayor competitividad económica y mayor calidad de vida, las decisiones se toman en un ambiente flexible que requiere el proceso político para abordar problemas complejos en un entorno cambiante y con alto grado de incertidumbre en contraposición a las rigideces de los planes urbanos. En este sentido, la planificación estratégica puede establecer un puente entre las rigideces del marco legal de la planificación urbana y, las decisiones políticas que demandan flexibilidad, ya que se concentra en temas críticos, esta orientada a la acción, promueve y fo- menta la participación de todos los agentes urbanos y estimula la modernización de la administración. La nueva cultura estratégica demanda tanto de los políticos como de los técnicos, un abordaje poco burocrático y capacidad para coordinar y dinamizar las fuerzas de la ciudad. En el Cuadro 2 se presentan las diferencias entre la planificación estratégica de las empresas y de las ciudades.

En nuestra propuesta asumimos la planificación estratégica urbana porque resuelve los tres retos conceptuales y operativos identificados anteriormente, "la incertidumbre creada por el dinamismo del entorno, la creciente complejidad de los procesos urbanos" $y$, viabiliza la participación "de la diversidad de agentes que intervienen en el desarrollo urbano" (Fernández Guell, 1997). En relación con los agentes intervinientes, coincidimos con este autor cuando dice que, para poner en marcha un proceso de participación es necesario, "comprender sus intereses e involucrarlos en el proceso de planificación". En definitiva, un plan estratégico urbano debe ser capaz de dar cabi$\mathrm{da}$ a los diferentes agentes locales y, su-

Cuadro 1

Comparación entre empresa y ciudad

\begin{tabular}{lll}
\multicolumn{1}{c}{ Aspectos comparados } & \multicolumn{1}{c}{ En la empresa } & \multicolumn{1}{c}{ En la ciudad } \\
\hline Propiedad & Accionistas & Ciudadanos y empresas \\
Alta Dirección & Consejo de Administración & Gobierno Local - Alcaldías \\
Productos & Manufactura o Servicios & Servicios, Calidad de Vida, \\
& & Puestos de Trabajo \\
Clientes & Consumidores & Ciudadanos, Inversores, \\
& & Turistas \\
Competidores & Otras Empresas & Otras Ciudades \\
\hline
\end{tabular}

Fuente : A partir de Fernández Gúell, 1998: 54. 


\section{Cuadro 2}

Diferencia entre la planificación estratégica de empresas y de ciudades

\begin{tabular}{|c|c|c|}
\hline Aspectos comparados & En la empresa & En la ciudad \\
\hline Definición de la Misión & Descripción Corta & Descripciốn compleja \\
\hline Nivel de Consenso & $\begin{array}{l}\text { Fuertemente Controlado - } \\
\text { Intervienen pocas personas }\end{array}$ & $\begin{array}{l}\text { Poco Controlado - Intervienen } \\
\text { muchas personas }\end{array}$ \\
\hline $\begin{array}{l}\text { Nivel de Exposición Pública } \\
\text { a través de los Medios }\end{array}$ & Baja & Alta \\
\hline Alcance del Plan & Fácil de definir & $\begin{array}{l}\text { Difícil de definir - Múltiples con- } \\
\text { sideraciones }\end{array}$ \\
\hline Evaluación de Alternativas & $\begin{array}{l}\text { Indicadores objetivos } \\
\text { y cuantitativos }\end{array}$ & $\begin{array}{l}\text { Indicadores Subjetivos y Cuali- } \\
\text { tativos }\end{array}$ \\
\hline Grado de Complejidad & Media & $\begin{array}{l}\text { Alta, con un componente iner- } \\
\text { cial muy fuerte, requiere de mu- } \\
\text { cho tiempo para cambiar el } \\
\text { rumbo de las ciudades }\end{array}$ \\
\hline Disponibilidad de Recursos & $\begin{array}{l}\text { Flexibilidad para asignar } \\
\text { recursos }\end{array}$ & $\begin{array}{l}\text { Gran rigidez para asignar } \\
\text { recursos }\end{array}$ \\
\hline
\end{tabular}

Fuente: A partir de Fernández Gúell, 1998: 57.

pralocales, que intervienen en los procesos de desarrollo urbano $y$, conciliar sus intereses, a partir de la formulación de una estrategia consensuada de futuro para la comunidad.

En este sentido y, en relación con nuestra propuesta de organizar a la comunidad, hay experiencias similares en otras ciudades como es el caso de la ciudad de Bilbao (España), donde la magnitud del proyecto de marketing de la ciudad, tanto a nivel nacional como internacional hizo necesario la creación de un organismo para aunar los distintos aportes, "coordinando todas las entidades e intereses implicados en el impulso del renacimiento del Bilbao Metropolitano. Esta necesidad cristalizó, "en una experiencia pionera de cooperación interinstitucional, con la creación de la Sociedad Pública Bilbao Ría 2000", (Bilbao, 1996). En el caso del
Plan de Gestión de Tamare se propone la creación de dos estructuras organizativas, una temporal el Comité de Gestión y otra permanente, FUNDATAMARE.

\subsection{La gestión urbana}

En relación con la gestión urbana, Fernández Güell identifica seis (6) cambios contextuales, la descentralización de la competencia urbanistica a los gobiernos locales, la creciente demanda de participación de los movimientos sociales, la incorporación de los agentes económicos en la gestión urbana, la creciente competitividad entre las ciudades para atraer inversiones nacionales y extranjeras (marketing urbano), la incorporación de innovación tecnológica en la gestión urbana como los Sistemas de Información Geográfica, (SIG), la automatización 
de los procesos administrativos y la mayor exigencia de transparencia en estos procesos. Según Precedo (1993 y 1996), el marketing urbano " es un modelo avanzado de planificación territorial e instrumento de gestión de las ciudades con enfoque empresarial cuyo objetivo es mejorar la productividad urbana". El marketing urbano asume la promoción de todos los aspectos que contribuyen al bienestar económico y a mejorar la calidad de vida en las ciudades.

En relación con la conceptualización de la gestión urbana, Merelo (1995:1) plantea que, "la gestión urbanistica en sentido amplio, engloba toda la actividad, esencialmente administrativa, sin perjuicio de la colaboración privada, tendente a desarrollary aplicar la ordenación urbanistica (no sólo el planeamiento urbanistico), en su doble vertiente de fomento o impulso y de control, en sentido estricto, para este autor, "la gestión urbanistica puede hacerse coincidir con la ejecución del planeamiento". El Ayuntamiento de Madrid (1996), asume como concepto amplio de gestión urbana, "el proceso completo de producción de lo urbano, que se inicia con la formulación, redacción, tramitación y aprobación de los Planes Urbanos y se desarrolla con su ejecución" $y$, define la gestión urbana en sentido estricto, como "la ejecución del Plan Urbano".

El Plan de Gestión de Tamare que proponemos, asume el concepto amplio de gestión urbanística por cuanto su objetivo es viabilizar la aprobación y ejecución del Plan Maestro de Desarrollo Urbano de Tamare, a través de la formulación consensuada del Convenio de Gestión.
Los planes para poder ejecutarlos y en consecuencia para que puedan incidir en la transformación y construcción de la ciudad, tienen que cumplir con tres requisitos:

- Que exista la Legislación, Ley Orgánica de Régimen Municipal (LORM) y Ley Orgánica de Ordenación Urbanistica (LOOU).

- Que exista la Capacidad de Gestión (Legal, Administrativa y Técnica).

- Que exista concordancia con los intereses y posibilidades de gestión de los Actores Sociales involucrados en la gestión de Tamare.

En este caso, el Plan Maestro de Desarrollo Urbano de Tamare cumple con los dos primeros requisitos, es un Plan Especial aprobado por la Alcaldía del Municipio Lagunillas, Institución Político Administrativa con capacidad de gestión legal, representada por existencia de las Ordenanzas de Ubanismo y Diseño Urbano del Plan Especial o Plan Maestro, aprobadas por la Cámara Municipal (instrumento legal) y técnica, representada por el equipo técnico de la Oficina Municipal de Planificación Urbana (OMPU) que esta preparado para implementarlo. El Plan de Ges. tión pretende lograr fundamentalmente el tercer requisito, la concordancia entre las propuestas del Plan y los intereses y posibilidades de gestión de los actores urbanos vinculados al Plan Maestro para que lo asuman como su proyecto y se comprometan con su ejecución.

Para la CEPAL (1995), "la gestión urbana es el conjunto de procesos dirigidos a operar sobre la ciudad, es la articulación de recursos humanos, financieros, organizacionales, politicos y naturales para construir la ciudad y satisfacer las necesi- 
dades individuales y colectivas de los distintos sectores de la población". El objetivo fundamental de la gestión urbana es mejorar la calidad de vida y, el bienestar de los ciudadanos a partir del incremento de la productividad y eficiencia en la prestación de los senvicios urbanos. Esto implica asumir la ciudad como espacio competitivo, de consumo y gestión, con una imagen (marketing urbano) que sirve para atraer inversiones nacionales e internacionales (Precedo, 1993).

\section{Propuesta metodológica para construir la viabilidad de los planes urbanos}

En este punto se describe la metodología que proponemos para elaborar el Plan de Gestión de Tamare, el cual tiene los siguientes objetivos generales y específicos.

\section{Objetivos Generales y Especificos del Plan de Gestión}

El Plan de Gestión de Tamare tiene dos objetivos generales:

- Construir la viabilidad, política, institucional, social y financiera, al Plan Maestro de Tamare.

- Organizar a la comunidad y a los actores con competencia en Tamare para que participen en la construcción de consensos y hacer de Tama. re un hábitat de calidad en la COLM.

Los objetivos específicos planteados son cuatro:
- Consolidar el desarrollo urbano de Tamare.

- Viabilizar el proceso de transferencia de competencias urbanas al Municipio.

- Consensuar y ejecutar el Convenio de Gestión.

- Conformar las estructuras organizativas para construir la viabilidad al Plan y ejecutar el Convenio de Gestión.

El Plan de Gestión propuesto se estructura en tres Momentos, el Momento 1: Construcción del Mensaje, Momento 2: Organización de los actores y el Momento 3: Construcción del Consenso o estrategia operativa (Cuadro 3 ).

\subsection{Momento 1: construir el mensaje y elaborar el Convenio de Gestión}

Un primer objetivo de este momento es construir el mensaje para explicar el Plan Maestro a los actores urbanos. EI mensaje inicial se elabora a partir de la artículación de dos productos: el Plan Maestro de Desarrollo Urbano de Tamare y los resultados de la encuesta realizada a diferentes actores, sobre la calidad de vida en Tamare. El mensaje se construye durante el proceso de viabilización y se concreta con la firma del Convenio de Gestión.

\section{a. El contenido del mensaje}

El objeto del mensaje es "explicar viabilizando" el Plan Maestro de Tamare, que exige la aplicación de una estrategia de marketing urbano y desarrollar el sentido de identidad y pertenencia en la 


\section{Cuadro 3}

Momentos del plan de gestión de tamare, objetivos y actores

\begin{tabular}{|c|c|c|}
\hline Momentos & Objetivos & Actores \\
\hline Momento 1 & $\begin{array}{l}\text { - Construir el mensaje para explicar - viabilizar el Plan } \\
\text { Maestro de Tamare. } \\
\text { - Elaborar el Convenio de Gestión. }\end{array}$ & $\begin{array}{l}\text { - LUZ-F.A. } \\
\text { - ASOVET*. }\end{array}$ \\
\hline Momento 2 & $\begin{array}{l}\text { - Seleccionar Actores. } \\
\text { - Conformar las estructuras organizativas (temporal y } \\
\text { permanente) para viabilizar y ejecutarel Plan Maestro. }\end{array}$ & $\begin{array}{l}\text { - LUZ-F.A. } \\
\text { - ASOVET. }\end{array}$ \\
\hline Momento 3 & $\begin{array}{l}\text { - Construir la viabilidad al Plan, política, institucional, so- } \\
\text { cial y económico - financiera. } \\
\text { - Conformar una platatorma de opinión favorable para } \\
\text { que los actores se comprometan con el Plan. } \\
\text { - Instalar FUNDATAMARE. } \\
\text { - Firmar el Convenio de Gestión. }\end{array}$ & $\begin{array}{l}\text { - Comité de Gestión } \\
\text { de Tamare. }\end{array}$ \\
\hline
\end{tabular}

Fuente: Elaboración propia.1997. *ASOVET : Asociación de vecinos de Tamare.

comunidad de Tamare. El contenido del mensaje tiene cuatro características básicas para lograr el compromiso de los actores:

- Abierto, es decir, ajustable en base a las sugerericias y propuestas consensuadas de los actores participantes para permitir la negociación entre agentes sociales y la toma de decisión compartida. El mensaje se construye con los actores durante el proceso de viabilización. Este planteamiento debe explicitarse en las reuniones de viabilización del Plan.

- Direccionado, el mensaje se formulará considerando las características y posicionamiento de los actores con respecto a Tamare y destacará aquellos objetivos y propuestas del Plan, que puedan ser compartidas o potencialmente negociables entre los actores y que por lo tanto servirán de apoyo durante el proceso de via- bilización del Plan (marketing - encuesta de opinión).

- Bidireccional, el proceso comunicacional desarrollado a partir de la difusión del mensaje permitirá la retroalimentación y el dialogo entre los actores, aspectos fundamentales para construir el mensaje consensualmente y así lograr el compromiso, de los actores, con el Plan Maestro.

- Entendible por todos los actores involucrados, se utilizará un lenguaje común que todos puedan entender. Su contenido debe dejar claramente explicado y fundamentado el Plan Maestro de Tamare y los escenarios alternativos si no se concreta el Plan.

\section{b. Estructura del Mensaje}

La explicación del Plan Maestro se apoya en el Sistema de Información Geográfico (SIG) de Tamare y se estructura de la forma siguiente: 
- Visión de Tamare: el Plan Maestro de Desarrollo Urbano de Tamare.

- Situación Actual de Tamare (problemas identificados a través de encuesta).

- Escenarios alternativos de Gestión (proceso de comunicación y retroalimentación).

- Convenio de Gestión: Tamare un Hábitat de Calidad en la COLM (compromiso de los actores).

La Visión de Tamare coincide con la propuesta urbana planteada en el Plan Maestro de Desarrollo Urbano de Tamare $y$, se construye, a partir de la sintesis descriptiva del Programa de Actuaciones Urbanísticas del Plan antes mencionando. En esta síntesis se destacan los aspectos clave, físico - espaciales (imagen), de equipamiento, infraestructurales $y$, socio - económicos, que caracterizan y apoyan el logro de una mejor calidad de vida en Tamare.

Por otro lado, para conocer la situación actual de Tamare en relación con la calidad de vida y, las expectativas de los diferentes actores sobre el "potencial de desarrollo" de Tamare, se diseñaron y aplicaron tres tipos encuesta.

- Encuesta dirigida a los Residentes Actuales de los 3 sectores del Lote Este, Urdaneta, Carabobo y Andrés Bello.

- Encuesta dirigida a los Residentes Potenciales (interesados en residir en Tamare).

- Encuesta dirigida a los Promotores Potenciales (interesados en construir Tamare).
La Encuesta tiene por objeto identificar las expectativas y opiniones de los residentes actuales y potenciales con respecto a la calidad de vida en Tamare $y$, de promotores privados y comerciantes interesados en desarrollar las áreas residenciales o comerciales vacantes de dicha urbanización. Conocer la opinión de los actores sobre Tamare servirá para direccionar el mensaje y para diseñar fórmulas viables de gestión que se incluirán como escenarios alternativos. La selección de la alternativa de gestión resultará de una evaluación ponderada y negociable, que se presentará a consideración de los actores sociales (quién gana y quién pierde y con qué alternativa ganan todos o casi todos) para la construcción de consensos.

Los escenarios alternativos de gestión se refieren a las distintas posibilidades de desarrollo de las áreas vacantes de Tamare y a la recuperación del uso de edificaciones existentes según lo planteado en el Plan. La formulación y selección preliminar de los escenarios o propuestas alternativas de gestión resultará de la contrastación de la encuesta con la situación objetivo esperada al consolidarse el Plan Maestro. El Plan Maestro viabilizado se corresponde con la imagen objetivo deseable y posible o VISIÓN de Tamare a largo plazo. Las diferentes alternativas de gestión se evaluarán considerando tres factores:

- Minimizar posibles impactos negativos.

- Las expectativas de los actores seleccionados (conocidas a través de encuesta). 
- La potencialidad de la alternativa para generar el apoyo y compromiso de los actores.

El Plan de Gestión incluye la firma de un Convenio de Gestión que concreta el compromiso y la corresponsabilidad de los agentes sociales con el éxito del Plan de Maestro de Tamare. El Convenio es inicialmente el Programa de Actuaciones Urbanísticas (PAU, Art.29 de la LOOU) del Plan Maestro de Tamare que se complementa y termina de construir con la participación de los actores seleccionados durante el proceso de viabilización del Plan Maestro. El Convenio definitivo incluirá las acciones establecidas en el PAU y las acciones acordadas con los actores para resolver los problemas identificados en la encuesta. El Convenio de Gestión incluye tres tipos de acciones:

- Acciones de tipo organizativo como la conformación de las estructuras organizativas, temporal y permanente que operativizan el Plan Maestro y el Convenio de Gestión respectivamente $y$, de carácter político administrativo, la constitución de la Parroquia Tamare.

- Acciones requeridas para consolidar Tamare, relacionadas con el mejoramiento y dotación de equipamiento urbano, tanto las que aparecen en el PAU como las planteadas por los actores durante el proceso de viabilización.

- Acciones de mejoramiento y dotación de servicios de infraestructura tanto las del PAU como las planteadas por los actores durante el proceso de viabilización.
Se priorizarán aquellas acciones cuya realización depende de la capacidad de gestión municipal y de los actores seleccionados: accesibilidad interior del transporte, nuevas áreas residenciales de calidad, mejora de áreas recreacionales y, la promoción de Tamare a nivel municipal-subregional y regional, para atraer inversiones.

\subsection{Momento 2: Organización de los actores}

El objetivo principal de este momento es estimular y promover la capacidad de los actores de Tamare para participar activamente, organizarse y cooperar, es decir potenciar la conformación de su capital social (Fukuyama, 1996). Para lograr este objetivo se conformaron dos Estructuras Organizativas, una temporal y otra permanente, que se encargarán de operativizar la estrategia del Plan de Gestión. La estructura organizativa temporal garantiza la viabilidad del Plan Maestro y, la estructura permanente, ejecuta el Convenio de Gestión. Este momento incluye la realización de las actividades siguientes:

- Caracterización y selección de actores.

- Conformación de la Estructura Organizativa Temporal.

- Conformación de la Estructuras Organizativa Permanente

\section{a. Caracterización y selección de actores}

Conocer y evaluar las caracteristicas de los actores con competencia en la 
gestión del Plan Maestro (quién está a favor, en contra o potencialmente a favor) es básico para direccionar el mensaje y para que se involucren en el proceso y se comprometan, es decir, para lograr una participación activa y construir la viabilidad al Plan.

- En el caso del Alcalde, se incluirán objetivos de su plan de gobierno aceptables por los demás actores participantes como: maximizar los impactos positivos, económicos y sociales, que conlleva, la consolidación de Tamare, sobre la población de Ciudad Ojeda; consolidar y mejorar la calidad de vida y ambiental del Municipio, promover el desarrollo de proyectos residenciales de calidad y formar parte y liderizar una actuación pionera y exitosa a nivel de la COLM.

- En el caso de las Asociaciones de Vecinos de Tamare (ASOVET), se considerarán sus objetivos como residentes de Tamare, entre estos, mantener y mejorar la calidad de vida. Esto implicaría consolidar la construcción del Plan de Tamare como esta previsto, es decir apoyar las iniciativas de LAGOVEN, participar en el diseño de políticas de mantenimiento, vigilancia y control del desarrollo y destacar los beneficios como generación de economías de escala y plusvalías. En este sentido, la participación la asumimos como el elemento clave que estructura toda nuestra propuesta y que va más allá del modelo donde el sujeto convocado a participar es el de "participante administrativo, o control de los consumidores" (Cunill, 1997: 130), en nuestra propuesta la participación ciudadana incluye la consulta para la formulación y desarrollo de políticas.

- En el caso de los residentes potenciales, Asociaciones Civiles, plantear como objetivos: mejorar sus condiciones de vida, vivir en un hábitat de calidad, entre otros.

Para seleccionar los actores que constituirán el Comité se consideraron tres aspectos (Ferrer, 1995): los objetivos de la Estrategia Operativa, las competencias asignadas en la legislación vigente, la actividad o función que realizan los actores o podrían realizar en el ámbito de la ejecución del Plan: deciden, planifican, financian, ejecutan, mantienen y/o controlan y los criterios siguientes:

- Competencia y/o interés en el Proyecto.

- Responsabilidades politicas.

- Responsabilidades gremiales.

- Representación de sectores económi$\cos$, sociales y culturales de la ciudad.

- Dirigentes de Juntas Parroquiales y de Vecinos.

- Nivel Técnico (asesores).

\section{b. Conformación de las estructuras} organizativas, temporal y permanente

Una de las causas principales de las dificultades que confrontan las Instituciones para viabilizar la concreción de sus planes y proyectos, es la ausencia de una estructura organizativa coherente que se responsabilice del proceso de gestión y de canales reglados que permi- 
tan optimizar la participación sinérgica de los actores.

El objetivo del Comité de Gestión es lograr que los actores interesados o no, se involucren y participen en la formulación y gestión del Proyecto. El Comité es un mecanismo participativo temporal (desaparece al constituirse la estructura permanente que lo absorbe) que integra los procesos de viabilización-gestión del Plan Maestro y facilita el diálogo y la participación sinérgica institucional y ciudadana para lograr el compromiso explícito de los actores con el Plan. Esta propuesta implica:

- Compartir responsabilidades y lograr el compromiso entre la comunidad, LAGOVEN, la Alcaldia, y los demás actores urbanos para consolidar el Plan Maestro.

- La necesidad de desarrollar nuevos enfoques y metodologías de gestión participativa.

Entre los objetivos del Comité de Gestión se encuentran:

- Viabilizar el Plan Maestro.

- Establecer mecanismos y canales organizados de participación.

- Construir los consensos y articular intereses entre los distintos actores.

- Establecer patrones de conducta nuevos que inducirán a la participación y a la toma de decisiones en situación de compromiso y cooperación (marketing e intervención social).

El proceso de viabilización permite la negociación entre diversos participantes para seleccionar por consenso las vías para resolver los problemas y facili- tar el diálogo, la comunicación y el compromiso entre la comunidad, LAGOVEN, la Municipalidad y las instituciones involucradas.

La selección de los actores para conformar el Comité de Gestión se realizó considerando dos aspectos : su vinculación con el Plan Maestro y su capacidad para desempeñar las funciones requeridas en los términos siguientes: responsabilidad (LAGOVEN, Alcaldía, ASOVET, Junta Parroquial), interés (comunidades organizadas, líderes políticos, comunitarios, gremiales, religiosos) poder (político, financiero), capacidad (técnica, económica) y aliados del Plan. La Facultad de Arquitectura lideriza el proceso y coordina el Comité (Cuadro 4).

\section{Conformación de la estructura organizativa permanente: FUNDATAMARE}

Se propone crear una Fundación, FUNDATAMARE, que se encargará de la ejecución del Convenio de Gestión. La Fundación estará integrada por los actores que conforman el Comité de Gestión y por un representante de ASOVET.

\subsection{Momento 3: construcción del consenso (diseño de la estrategia operativa)}

El objeto de la estrategia operativa es construir la viabilidad al Plan (politica, institucional, social y económico financiera) y conformar una plataforma de opinión favorable, para que los actores involucrados se comprometan con el Plan y lo asuman como su proyecto. 
Cuadro 4

Identificación de actores del comité de gestión

\begin{tabular}{|c|c|c|c|c|c|c|}
\hline Viabilidad & & Política & & Institucional & $\begin{array}{l}\text { Social - Cultural } \\
\text { (Sociedad } \\
\text { Organizada) }\end{array}$ & $\begin{array}{c}\text { Econ-Financiero } \\
\text { (Promotores) }\end{array}$ \\
\hline A & • & Alcalde & - & LAGOVEN & - ASOVET. & - $\quad \mathrm{ACIL}^{*}$ \\
\hline C & & $\begin{array}{l}\text { Concejal } \\
\text { del circuito }\end{array}$ & $\bullet$ & OMPU, OMTTU & $\begin{array}{l}\text { Asociaciones } \\
\text { Civiles }\end{array}$ & - Construcción \\
\hline $\mathrm{T}$ & & $\begin{array}{l}\text { Junta Parroquial } \\
\text { Libertad }\end{array}$ & - & Ing. Municipal & $\begin{array}{l}\text { Asoc. Civil } \\
\text { La Pradera. }\end{array}$ & - Inmobiliaria \\
\hline 0 & $\bullet$ & $\begin{array}{l}\text { Líderes } \\
\text { Políticos }\end{array}$ & - & ENELCO & - Párroco & - Comercio \\
\hline $\mathrm{R}$ & & & $\bullet$ & IMAULA & $\begin{array}{l}\text { Dir. Escuela } \\
\text { Fray Luis }\end{array}$ & - Promotores \\
\hline$E$ & & & • & HIDROLAGO & - Gremios & - Contratistas \\
\hline $\mathrm{s}$ & & & • & $\begin{array}{l}\text { LAGUNIGAS } \\
\text { FALUZ }\end{array}$ & & \\
\hline
\end{tabular}

Fuente: Elaboración propia 1997. * ACIL: Asociación de Comerciantes e industriales de Lagunillas.

Para lograr estos objetivos se propone desarrollar un proceso democrático de participación (reuniones) que permitirá tomar decisiones en correspondencia con las demandas sociales. Esta estrategia facilitará la construcción de consensos, el desarrollo de un proceso de aprendizaje social y, el cambio de actitud de los actores con respecto al Plan Maestro, durante el proceso de participación y debate que se llevara a cabo para viabilizar y consolidar el desarrollo del Plan Maestro.

La estrategia operativa que se propone se desarrolla en tres etapas (Cuadro 5):

- La Etapa 1: Compromiso de participación, que se corresponde con la instalación del Comité de Gestión.
- La Etapa 2: Marketing o mercadeo de Tamare.

- La Etapa 3: Corresponsabilidad en la Gestión, firma del Convenio de Gestión e instalación de FUNDATAMARE.

a. Etapa 1: Compromiso de Participación.

El objetivo de esta etapa es instalar el Comité de Gestión para construir la viabilidad del Plan Maestro de Tamare.

b. Etapa 2: Marketing de Tamare.

El proceso de viabilización se inicia con la divulgación del mensaje o explicación del Plan Maestro a los agentes urbanos a partir de la aplicación de métodos de promoción urbana o marketing urba- 
Cuadro 5

Estrategia operativa plan de gestión de Tamare

\begin{tabular}{|c|c|c|}
\hline Etapas & Objetivos & Actores \\
\hline \multirow[t]{2}{*}{$\begin{array}{l}\text { Etapa 1: Compromiso de parti- } \\
\text { cipación. }\end{array}$} & $\begin{array}{l}\text { Instalar el Comité } \\
\text { de Gestion. }\end{array}$ & $\begin{array}{l}\text { - LUZ-F.A, ASOVET, OMPU, } \\
\text { Concejal, Párroco, IMAULA }\end{array}$ \\
\hline & & $\begin{array}{l}\text { HIDHOLAGO, ENELCO, } \\
\text { LAGUNIGAS, La OMPU, } \\
\text { OMDS, OMTTU e Ingenie- } \\
\text { ría Municipal, Junta Parro- } \\
\text { qulal. }\end{array}$ \\
\hline Etapa 2: Marketing de Tamare. & $\begin{array}{l}\text { Aplicar el marketing } \\
\text { a Tamare. }\end{array}$ & $\begin{array}{l}\text { - Comité de Gestión de Ta- } \\
\text { mare. }\end{array}$ \\
\hline $\begin{array}{l}\text { Etapa } 3 \text { : Corresponsabilidad } \\
\text { en la Gestión. }\end{array}$ & $\begin{array}{l}\text { Firmar el Convenio } \\
\text { de Gestión. } \\
\text { Instalar FUNDATAMARE. }\end{array}$ & $\begin{array}{l}\text { - Comité de Gestión de Ta- } \\
\text { mare. }\end{array}$ \\
\hline
\end{tabular}

Fuente: Ferrer y Avila, elaboración propia, 1997.

no. Los aspectos que caracterizan a Tamare y que forman parte de la estrategia de marketing son los siguientes:

1. La imagen atractiva de Tamare tanto para los usuarios actuales y como para los potenciales, como modelo de urbanización petrolera abierta, zona residencial por excelencia, con buena dotación de servicios y calidad ambiental, factores muy positivos que la caracterizan.

2. Las ventajas comparativas de Tamare para atraer residentes e inversores, considerando su calidad ambiental y de vida. La calidad medioambiental de Tamare y su oferta de áreas residenciales de calidad, servicios de equipamiento, áreas de trabajo y comercio, es actualmente y será a futuro con la apertura petrolera un atractivo importante que podrá determinar su elección como lugar de residencia de la mano de obra más calificada, lo que constituye un fuerte factor de atracción para la localización de empresas. El éxito obtenido por Tamare para atraer inversionistas tendrá un impacto positivo en el Municipio Lagunillas (mayores impuestos) y en consecuencia en Ciudad Ojeda.

3. La práctica en Tamare de una eficiente gerencia urbana, realizada por LAGOVEN, que ha permitido la implantación de políticas de cooperación pública-privada y pública-pública y ha generado los cambios requeridos en el tejido social de Tamare, que facilitarán la viabilidad del Plan.

4. La selección y utilización de una consigna que refleja lo que se quiere lograr con el Plan de Gestión y que pueda movilizar y motivar a los actores a actuar. "Tamare, Un Hábitat de Calidad en la COLM". 
El objetivo general de la estrategia de marketing dentro de esta práctica es promover interrelaciones entre los que viven y desarrollan su actividad en Tamare: ciudadanos, empresas, instituciones y otros agentes locales y los actores con competencia en el desarrollo y consolidación urbana de Tamare: la Alcaldia, las Instituciones prestadoras de servicios, entre otros. Con este enfoque la planificación urbana deja de ser un modelo desarrollista para convertirse en un modelo de calidad, siendo los aspectos cualitativos los que diferencian ambos modelos. Sus contenidos y objetivos concuerdan con las aspiraciones y problemas detectados en Tamare a través de la encuesta y son los siguientes:

- Mejorar la calidad de la vivienda, de las áreas residenciales, parques y canchas deportivas.

- Mejorar las infraestructuras de transporte público y telecomunicaciones.

- Incrementar el poder político de Tamare creando la Parroquia Tamare.

- Viabilizar el incremento del presupuesto municipal para ejecutar la política de renovación urbana que requiere Tamare y exige disponibilidad financiera de la Alcaldía. c. Etapa 3: Ejecución de la Estrategia operativa.

Para construir la viabilidad al Plan Maestro de Tamare se realizaron una serie de reuniones con el Comité de Gestión para dar a conocer el Plan de Gestión de Tamare, formular el Convenio de Gestión $e$ instalar FUNDATAMARE. Se planificaron inicialmente cuatro reuniones como se indican en el Cuadro 6.

El proceso de viabilización finaliza con la instalación de FUNDATAMARE y la firma del Convenio de Gestión, a través del cual se concreta la alternativa seleccionada de gestión y la alianza estratégica entre los actores. El Plan de Gestión, configurado a partir del principio de participación y compromiso de los actores involucrados, ofrece un Modelo de Gestión que tiene otros efectos positivos en la medida que actúa como marco de referencia que induce a otros actores a iniciar proyectos o estrategias que complementen y faciliten la consecución de los objetivos planteados. Esta confluencia de esfuerzos y acciones sinérgicas, iniciadas desde los sectores público y privado, garantizan el éxito del Plan de Gestión de Tamare. 


\section{Cuadro 6}

Ejecución de la estrategia operativa, reuniones, objetivos y actores - plan de gestión de Tamare

\begin{tabular}{|c|c|c|}
\hline Reuniones & Objetivos & Actores \\
\hline Reunión Inicial & $\begin{array}{l}\text { - Lograr la aprobación del Plan de Gestión pro- } \\
\text { puesto. } \\
\text { - Solicitar la designación de uno o más represen- } \\
\text { tantes para participar en el Comité de Gestión. }\end{array}$ & $\begin{array}{l}\text { - LUZ, F. Arq. y LA- } \\
\text { GOVEN. } \\
\text { - Instituciones acto- } \\
\text { res públicos y pri- } \\
\text { vados selecciona- } \\
\text { dos. }\end{array}$ \\
\hline Primera Reunión & $\begin{array}{l}\text { - Instalar el Comité de Gestión. } \\
\text { - Informar sobre el Plan de Gestión y cronograma } \\
\text { de reuniones. } \\
\text { - Lograr el compromiso inicial de estos actores con } \\
\text { el Plan de Gestión. } \\
\text { - Elaborar la minuta con los acuerdos de la reunión } \\
\text { y la agenda de la tercera reunión. }\end{array}$ & $\begin{array}{l}\text { - LUZ-F.A, ASO- } \\
\text { VET, OMPU, Con- } \\
\text { cejal, Párroco, } \\
\text { IMAULA HIDRO- } \\
\text { LAGO, ENELCO, } \\
\text { LAGUNIGAS, } \\
\text { OMPU, OMDS, } \\
\text { OMTTU, Ingeniería } \\
\text { Municipal, Junta } \\
\text { Parroquial. }\end{array}$ \\
\hline Segunda Reunión & $\begin{array}{l}\text { - Explicar el Plan Maestro de Tamare. } \\
\text { - Establecer los primeros compromisos: acepta- } \\
\text { ción de la visión, escenarios alternativos, Conve- } \\
\text { nio y Fundatamare } \\
\text { - Elaborar la minuta con los acuerdos de la reunión } \\
\text { y la agenda de la cuarta reunión. }\end{array}$ & - Comitê de Gestión. \\
\hline Tercera Reunión & $\begin{array}{l}\text { - Viabilizar y concretar el Convenio de Gestión. } \\
\text { Elaborar las minutas con los acuerdos logrados y } \\
\text { las agendas que deberán cumplirse hasta con- } \\
\text { cretar la firma del Convenio de Gestión y la insta- } \\
\text { lación de FUNDATAMARE. }\end{array}$ & - Comité de Gestión. \\
\hline
\end{tabular}

Fuente: Elaboración propia, 1997.

\section{Bibliografía citada}

Ayuntamiento de Madrid (1996). Memoria, Plan General de Ordenación Urbana Madrid. Eurocolor, S.A..

Bilbao, Ria 2000 (1996). Memorla. BSB Publicidad S.A. Bilbao. España.

CEPAL (1995). Gestión Urbana en Ciudades intermedias. Documento.
Cunill, Nuria (1997). Repensando lo Público a través de la Sociedad. CLAD. Editorial Nueva Sociedad. Caracas.

Fernández Guell, José Miguel (1997). Planificación Estratégica de Ciudades. Editorial Gustavo Gili, S.A.

Ferrer, Mercedes (1993). La Gestión Urbana: Un nuevo Modelo para la construcción de la Ciudad. Trabajo de Ascenso a profesor Asociado, Facultad de 
Arquitectura, Universidad del Zulia. Maracaibo.

Ferrer, Mercedes (1995). Modelo de Gestión:

La praxis de la construcción del Plan Municipal y la Ciudad. Trabajo de Ascenso a profesor Titular, Facultad de Arquitectura, Universidad del Zulia. Maracaibo.

Ferrer, Mercedes; Avila, Nancy et alt (1997). Plan de Gestión de Tamare, Fase II, Etapa 1 "Relaciones con la Comunidad". Convenio LUZ-LAGOVENALCALDIAS DE LA COLM-ACIVE. GESUR. Facultad de Arquitectura, LUZ. Maracaibo.

Fukuyama, Francis (1996). CONFIANZA (Trust). Las virtudes sociales y la capacidad para generar prosperidad. Editorial Atlantida. Buenos Aires.

Martinez, Luzmarina, Ferrer, Mercedes, Avila Nancy (1997). Plan de Gestión de Tamare, Fase II, Etapa 2. Estrategia para viabilizar el Plan Maestro de Tamare. Convenio LUZ-LAGOVEN-
ALCALDIAS DE LA COLM-ACIVE. GESUR. Facultad de Arquitectura, LUZ. Maracaibo.

Melo Moya, Sadi (1995). Una estrategia Rectoral para la Comuna de El Bosque. En, Colección Documentos Técnicos de la Comunidad de Madrid, Aplicación de la Intervención Estratégica en la Ciudad. Conserjeria de Política Territorial. Dirección General de Arquitectura. Madrid. España.

Merelo Abela, José Manuel (1995). Régimen Jurídico del Suelo y Gestión Urbanistica. Praxis S.A.

Precedo, Andrés (1993). "Las políticas de desarrollo y renovación urbana en Europa". En, Ciudad y Territorio - Estudios Territoriales, MOPU. Madrid. Vol. 1 (98).

Precedo, Andrés (1996). Ciudad y Desarrollo Urbano. Editorial Sintesis S.A. Madrid. 